\title{
Effects of Self-Esteem on Resilience and School Happiness of Korean Students Participating in School Sports Clubs ${ }^{\wedge}$
}

\author{
Hohyuns, Song ${ }^{1}$, Jihwan, Song ${ }^{2}$, Seungman, Lee ${ }^{3}$ \\ ${ }^{1}$ Jeonju Hosung Middle School, Republic of Korea \\ ${ }^{2}$ Jeonju National University of Education, Republic of Korea \\ ${ }^{3}$ Kyunghee University, Republic of Korea \\ 'Hohyunss@nate.com, ${ }^{2}$ ufosong114@jnue.kr, ${ }^{3} 1 \mathrm{sm} 14$ pe@khu.ac.kr
}

\begin{abstract}
School students can gain various advantages such as self-esteem and resilience through sports activities. This study examined the effects of self-esteem on resilience and school happiness of School students participating in school sports clubs. We aimed to provide primary data on the role of school sports clubs in the promotion of school happiness and how they can be used for this purpose. Through convenience sampling, this research selected around 324 Korean School students (84 for a preliminary and 240 for a primary survey) participating in school sports clubs. For data analyses, we used frequency, reliability and validity tests, descriptive statistics, correlation analysis, and multiple regression analysis. Results were: First, there was a significant correlation between self-esteem, resilience, and school happiness; second, positive self-esteem had positive effects on all subfactors of resilience, and negative self-esteem had negative effects on these same subfactors; third, positive selfesteem had positive effects on all subfactors of school happiness, and negative self-esteem had negative effects on some subfactors (student-teacher relationship, environmental satisfaction, learning activity pleasure, and psychological stability). Therefore, the development of ways to expand school sports clubs may help enhancing School students' self-
\end{abstract}

\section{Seungman, Lee}

Kyunghee University, Republic of Korea lsm14pe@khu.ac.kr esteem and resilience, so that students, who spend most of their time in school, can feel happy in this daily recurring setting.

Keywords : School students, School sports clubs, Self-esteem, Resilience, School happiness

\section{Introduction}

School students in South Korea spend most of their time in school, which does not keep pace with social changes and focuses only on entrance exam-oriented education to enter higher schools. This leads to excessive competition, which is why the pressure and stress that dominate School students are causing their problems. Also, School students lack physical activity, which leads to poor physical strength and obesity.To solve these problems, the Ministry of Education introduced the School Sports Club Policy in 2007 to provide students with sufficient physical activity. Unlike regular physical education classes, school sports clubs are meaningful in that students chose their own sports classes, so they can be more immersed in activities, thereby relieving anxiety, stress, and tension that can be common in School years, and improving their potential.

Generally speaking, living in a modern - and complex - society denotes that people are not able to avoid some stressful situations, which means that, rationally, people should try to adapt to these situations, and feeling happy in and with their lives may be crucial in this regard; with this in mind, we believe that education can promote such happiness 
because it has the potential to increase people's capacity to more flexibly adapt to internal and external stress factors. According to a variety of psychological studies on happiness and stress adaptation, people's capacity to cope with and adapt to stress depend on their personality traits and on social and environmental factors, which is the reason why in depth studies on personal trait factors are needed [12]. [3] analyzed variables affecting students' happiness, and they emphasized the importance of personality traits in happiness; these variables, along with economic status, presented main effects on happiness. As for Korean School students who spend most of their time at school, they were shown to feel happy in school [4]; this goes to show that researching personal trait factors affecting students' happiness while considering their school happiness is necessary.

Generally, school happiness refers to students' emotional wellness followed by psychological satisfaction caused by performing school activities [5]. This concept covers psychological factors present in the school setting, including friendship, studentteacher relationship, self-efficacy, environmental satisfaction, learning activity pleasure, and psychological stability. Moreover, negative emotions in school can lead to various common youth problems, such as school violence, school maladjustment, suicide, bullying, etc. [6]. Summarizing, school happiness, which is a positive emotion, is crucial for youth.

Physical activities, such as sports, have recently received much attention because they help promote school happiness, and the Korean government has introduced and implemented sports club programs in schools [7]. According to several studies on the effectiveness of school sports clubs, about $67 \%$ of adults who felt happy in School were more interested in sports activities and feel much happier in adulthood than those who had high academic achievement (3.5\%; [8]). Such findings indicate that their school experiences affect their happiness during adulthood.

An adolescent physical activity participation policy can influence psychological characteristics, one of which is self-esteem: a previous study showed that sports activity is the most important activity for adolescents to build confidence, which is essential for creating self-esteem, and adolescents participate in sports clubs to learn sports skills and to create a sense of self-concept and self-esteem in the midst of their interactions with peers [9]. This self-esteem is essential for the development of a healthy personality, adaptation to the environment, and self-realization [10], which then affects life positivity and satisfaction [11]. Further, it is essential for personal growth and development, and it is known to be highly related to personal happiness [12]. In some studies, people with high self-esteem were shown to have a positive identity about themselves, which then led to building higher resilience: when coping with unexpected crises, they tend to accept themselves without being influenced by negative evaluations from others [5], [13-14]. These studies showed that self-esteem can influence resilience.

Deepening this discussion, another significant variable in determining adolescents' happiness would be resilience, a concept that has been recently attracting much attention in many scientific fields. Resilience is a positive force that enables individuals to successfully overcome stress or difficulties and adapt to reality [15]. It can be defined as a composure by which people positively face and solve challenges while interacting with the environment [16]. When people are faced with the same stressful challenges, they show differences in stress relief and problemsolving ability owing to their different resilience capacities [17]; this same study adds that resilience is not an innate acquisition, but an ability that gradually forms and gets enhanced during one's development while dynamically interacting with the environment, that is, this ability can potentially be learned progressively through sports activities. Recent research has shown that proper physical activities and continuous exercise can promote resilience, which is a decisive ability to cope with challenges [18]. Another research indicates that it also affects academic achievement, school life satisfaction, happiness, and internal stability through the cultivation of core skills and attitudes, which allows for adolescents to grow and develop themselves in positive ways [19]. There are many previous studies on the relationship between adolescents' sports participation, self-esteem, resilience, school life adaptation, and psychological satisfaction. However, studies on the relationship between sports activity, self-esteem, resilience, and school happiness among samples of School students, who are a very vulnerable populational group to stress because they have to worry about university entrance and entering in the work market, remain scarce. Therefore, this study aimed to examine how selfesteem of School students participating in school sports clubs affects resilience and school happiness.

This study aimed to provide primary data on the role 
of school sports clubs in the promotion of school happiness and how they can be used for this purpose. Further, it aimed to help develop research and education programs for School students. With these intents, we produced three research hypotheses, as follows:

Hypothesis 1: the self-esteem of School students participating in school sports clubs would have an effect on resilience.

Hypothesis 2: their self-esteem would have an effect on school happiness.

Hypothesis 3: their resilience would have an effect on school happiness.

\section{Method}

\subsection{Sample}

We used convenience sampling for sample recruitment. The preliminary survey occurred in May 2019, and 84 students in the South and North Jeolla Province participated. And the main survey occurred in October 2019, and 240 students in Gwangju Metropolitan City participated. The general characteristics of the samples are shown in [Table 1].

\section{Table1: General Characteristics of the Sample}

\begin{tabular}{|c|c|c|c|c|c|}
\hline \multirow[b]{2}{*}{ Characteristics } & \multirow[b]{2}{*}{ Group } & \multicolumn{2}{|c|}{ Preliminary survey } & \multicolumn{2}{|c|}{ main survey } \\
\hline & & \begin{tabular}{|l}
$\begin{array}{l}\text { Frequency } \\
\text { (n) }\end{array}$ \\
\end{tabular} & \begin{tabular}{|l|}
$\begin{array}{l}\text { Composition rate } \\
(\%)\end{array}$ \\
\end{tabular} & $\begin{array}{l}\text { Frequency } \\
\text { (n) }\end{array}$ & \begin{tabular}{|l}
$\begin{array}{l}\text { Composition rate } \\
(\%)\end{array}$ \\
\end{tabular} \\
\hline \multirow{2}{*}{ Gender } & Male & 44 & 52.4 & 103 & 42.9 \\
\hline & Female & 40 & 47.6 & 137 & 57.1 \\
\hline \multirow{3}{*}{ Grade } & 1 & 34 & 40.5 & 87 & 36.3 \\
\hline & 2 & 29 & 34.5 & 99 & 41.3 \\
\hline & 3 & 21 & 25 & 54 & 22.5 \\
\hline \multicolumn{2}{|l|}{ Total } & 84 & 100 & 240 & 100 \\
\hline
\end{tabular}

\subsection{Instruments}

Questionnaires were used as survey tools. The questionnaires used in this research were selected based on their reliability and validity values, as they had already been proven in several previous researches. Questions on the general characteristics of the sample consisted of gender and grade. To measure participants' self-esteem, we used the translated version of the 10-item Rosenberg Self-Esteem Scale (RSES) by [20], developed by [21]. It has two subscales: positive (5 items) and negative self-esteem (5 items). To measure participants' resilience, we used the 27-item questionnaire developed by [22] and used by [23]. It consists of three subscales: control (9 items), positivity ( 9 items), and sociality (9 items). To measure participants' school happiness, we used the 22 -item Authentic Happiness Inventory Questionnaire suggested by [24], which was developed considering school situations by [5]. It has 6 subscales: friendship, student-teacher relationship, self-efficacy, environmental satisfaction, learning activity pleasure, and psychological stability.

\subsection{Reliability Of Scale}

Reliability refers to the degree of consistency of questions present in a measure, covering such concepts as response stability, consistency, and predictability [25]. There are several ways to measure reliability, but this study used the Cronbach's $\alpha$ coefficient. The Cronbach's $\alpha$ coefficient of the selfesteem measure ranged from .776-.893, that for the resilience measured range from .866-.894, and for the school happiness measure ranged from .441-.883. All subfactors of all measures showed Cronbach's $\alpha$ coefficients of .6 or higher, which exceeded the required threshold; nevertheless, friendship, one of the subfactors of the school happiness measure, showed a value of .441, so it was excluded from this research.

\subsection{Validity Of Scale}

Validity refers to how accurately the tool assesses the concepts or properties it allegedly measures, and the developed measurement tool should precisely reflect its corresponding properties [25]. To check for the validity of contents, we drafted a preliminary questionnaire based on previous research and reviewed it; for this purpose, we met with five experts in the field of sports, consisting of researchers and PhDs. Moreover, construct validity, convergent validity, and discrimination validity were verified through confirmatory factor analysis. The conformity factor analysis of the research model is shown in [Table 2].

\section{Table 2 : Conformity Factor Analysis Models}

\begin{tabular}{|l|l|l|c|c|c|c|c|c|c|l|}
\hline Model & $x^{2}$ & df & CMIN/DF & RMR & GFI & CFI & NFI & IFI & $\begin{array}{l}\text { RMSE } \\
\text { A }\end{array}$ & $\boldsymbol{p}$ \\
\hline Index & 334.142 & 129 & 2.59 & .045 & .863 & .938 & .903 & .938 & .082 & .000 \\
\hline $\begin{array}{l}\text { Conformity } \\
\text { criteria }\end{array}$ & - & - & $\leqslant 3.0$ & $\leqslant .05$ & $\geqslant .90$ & $\geqslant .90$ & $\geqslant .90$ & $\geqslant .90$ & $\leqslant .10$ & .000 \\
\hline
\end{tabular}

All models, except for the GFI model, were found to exceed conformity thresholds. The GFI model indicated a poor conformity, recoding less than .90 . However, this model can be affected by inconsistencies owing to sample characteristics, so it is considered that the CFI and RMSEA values, which 
are relatively not affected by these same inconsistencies, are essential criteria for determining conformity [26]. Summarizing, the relationship between variables was considered appropriate in this questionnaire.

Convergent and discriminant validity were verified through a confirmatory factor analysis (see Table 3). A questionnaire with convergent validity means that it has a high correlation between values that measure the same concept by different methods [25]. This validity can be confirmed through the use of three methods: the standardized coefficients of all variables must be higher than .5 while the C.R. values must be of at least 1.965; the variance distribution mean values must be of at least .5; or all construct reliability values must be higher than .7. We employed all three methods to confirm convergent validity, and results are shown in [Table 3 ].

Table 3 : Confirmatory Factor Analysis Result

\begin{tabular}{|c|c|c|c|c|c|c|c|}
\hline Factor & $\begin{array}{l}\text { Unstandardize } \\
\text { d coefficients }\end{array}$ & S.E. & C.R. & $p$ & $\begin{array}{l}\text { Standardize } \\
\text { d coefficients }\end{array}$ & $\begin{array}{l}\text { Mean } \\
\text { variance } \\
\text { distributio } \\
\mathbf{n}\end{array}$ & $\begin{array}{l}\text { Construct } \\
\text { Reliabilit } \\
\mathbf{y}\end{array}$ \\
\hline $\begin{array}{l}\text { Positive Self } \\
\rightarrow \text { Positive } \\
\text { Self } 7\end{array}$ & 1 & - & - & - & .884 & \multirow{5}{*}{.949} & \multirow{5}{*}{.991} \\
\hline $\begin{array}{l}\text { Positive Self } \\
\rightarrow \text { Positive } \\
\text { Self } 6\end{array}$ & .943 & $\begin{array}{c}.05 \\
3\end{array}$ & $\begin{array}{c}17.74 \\
3\end{array}$ & $\begin{array}{c}.000^{*} \\
*\end{array}$ & .852 & & \\
\hline $\begin{array}{l}\text { Positive Self } \\
\rightarrow \text { Positive } \\
\text { Self } 4\end{array}$ & .665 & $\begin{array}{c}.05 \\
1\end{array}$ & $\begin{array}{c}13.01 \\
1\end{array}$ & $\begin{array}{l}.000^{*} \\
*\end{array}$ & .709 & & \\
\hline $\begin{array}{l}\text { Positive Self } \\
\rightarrow \text { Positive } \\
\text { Self } 2\end{array}$ & .750 & .05 & $\begin{array}{c}14.26 \\
7\end{array}$ & $\begin{array}{c}.000^{*} \\
*\end{array}$ & .752 & & \\
\hline $\begin{array}{l}\text { Positive Self } \\
\rightarrow \text { Positive } \\
\text { Self } 1\end{array}$ & .773 & .05 & $\begin{array}{c}14.49 \\
3\end{array}$ & $\begin{array}{c}.000^{*} \\
*\end{array}$ & .759 & & \\
\hline $\begin{array}{l}\text { Negative Self } \\
\rightarrow \text { Negative } \\
\text { Self } 10\end{array}$ & 1 & - & - & - & .772 & \multirow{4}{*}{.929} & \multirow{4}{*}{.981} \\
\hline $\begin{array}{l}\text { Negative Self } \\
\rightarrow \text { Negative } \\
\text { Self } 9\end{array}$ & 1.023 & $\begin{array}{c}.08 \\
1\end{array}$ & $\begin{array}{c}12.64 \\
3\end{array}$ & $\begin{array}{c}.000^{*} \\
*\end{array}$ & .793 & & \\
\hline $\begin{array}{l}\text { Negative Self } \\
\rightarrow \text { Negative } \\
\text { Self } 5\end{array}$ & .983 & $\begin{array}{c}.07 \\
5\end{array}$ & $\begin{array}{c}13.15 \\
6\end{array}$ & $\begin{array}{c}.000^{*} \\
*\end{array}$ & .822 & & \\
\hline $\begin{array}{l}\text { Negative Self } \\
\rightarrow \text { Negative } \\
\text { Self } 3\end{array}$ & .904 & $\begin{array}{l}.07 \\
0\end{array}$ & $\begin{array}{c}12.86 \\
7\end{array}$ & $\begin{array}{c}.000^{*} \\
*\end{array}$ & .805 & & \\
\hline $\begin{array}{l}\text { Resilience } \rightarrow \\
\text { Sociality }\end{array}$ & 1 & - & - & - & .749 & \multirow{3}{*}{.977} & \multirow{3}{*}{.992} \\
\hline $\begin{array}{l}\text { Resilience } \rightarrow \\
\text { Positivity }\end{array}$ & 1.233 & $\begin{array}{c}.08 \\
9\end{array}$ & $\begin{array}{c}13.79 \\
9\end{array}$ & $\begin{array}{c}.000^{*} \\
*\end{array}$ & .848 & & \\
\hline $\begin{array}{l}\text { Resilience } \rightarrow \\
\text { Control }\end{array}$ & 1.226 & $\begin{array}{c}.09 \\
0\end{array}$ & $\begin{array}{c}13.56 \\
4\end{array}$ & $\begin{array}{c}.000^{*} \\
*\end{array}$ & .836 & & \\
\hline $\begin{array}{l}\text { School } \\
\text { happiness } \rightarrow \\
\text { Psychological } \\
\text { stability }\end{array}$ & 1 & - & - & - & .582 & \multirow{5}{*}{.953} & \multirow{5}{*}{.991} \\
\hline $\begin{array}{l}\text { School } \\
\text { happiness } \rightarrow \\
\text { Learning } \\
\text { activity } \\
\text { pleasure }\end{array}$ & 1.376 & $\begin{array}{c}.13 \\
7\end{array}$ & $\begin{array}{c}10.06 \\
5\end{array}$ & $\begin{array}{l}.000^{*} \\
*\end{array}$ & .890 & & \\
\hline $\begin{array}{l}\text { School } \\
\text { happiness } \rightarrow \\
\text { Environmenta } \\
\text { 1 satisfaction }\end{array}$ & 1.273 & $\begin{array}{l}.12 \\
8\end{array}$ & 9.974 & $\begin{array}{l}.000^{*} \\
*\end{array}$ & .876 & & \\
\hline $\begin{array}{l}\text { School } \\
\text { happiness } \rightarrow \\
\text { Self-efficacy }\end{array}$ & 1.213 & $\begin{array}{c}.12 \\
1\end{array}$ & $\begin{array}{c}10.03 \\
5\end{array}$ & $\begin{array}{c}.000^{*} \\
*\end{array}$ & .886 & & \\
\hline $\begin{array}{l}\text { School } \\
\text { happiness } \rightarrow \\
\text { Student- } \\
\text { teacher } \\
\text { relationship }\end{array}$ & 1.203 & $\begin{array}{c}.12 \\
5\end{array}$ & 9.651 & $\begin{array}{c}.000^{*} \\
*\end{array}$ & .828 & & \\
\hline
\end{tabular}

For the first method, results showed that the standardized coefficients of all variables were of at least .5 (.582-.890) while the C.R. values were higher than 1.965 (7.951-17.743), so convergent validity was confirmed. For the second method, results showed that all variance distribution mean values reached at least .5 (.929-.977), so convergent validity was supported. For the third method, all construct reliability values were higher than .7 (.981-.992), so convergent validity was confirmed once more. Thus, we assumed that the measures had appropriate convergent validity.

Discriminant validity means that the correlations between different constructs should have significance even with low values [25]. In this research, the correlation between constructs and variance distribution mean values were used to confirm discriminant validity. The discriminant validity test results are shown in [Table 4].

Table 4 : Correlation between constructs

\begin{tabular}{|l|c|c|c|c|c|}
\hline \multirow{2}{*}{ Variables } & \multicolumn{4}{|l|}{ Correlation between constructs } & $\begin{array}{l}\text { Mean } \\
\text { Variance } \\
\text { distribution }\end{array}$ \\
\cline { 2 - 6 } & $\begin{array}{l}\text { Positive Self- } \\
\text { esteem }\end{array}$ & $\begin{array}{l}\text { Negative Self- } \\
\text { esteem }\end{array}$ & Resilience & $\begin{array}{l}\text { School } \\
\text { Happiness }\end{array}$ \\
\hline $\begin{array}{l}\text { Positive Self- } \\
\text { esteem }\end{array}$ & 1 & - & - & - & .949 \\
\hline $\begin{array}{l}\text { Negative Self- } \\
\text { esteem }\end{array}$ & -.700 & 1 & - & - & .929 \\
\hline Resilience & .784 & -.675 & 1 & - & .977 \\
\hline School Happiness & .732 & -.642 & .855 & 1 & .953 \\
\hline
\end{tabular}

Since it is challenging to verify discriminant validity among all variables, researchers usually select and verify similar concepts or a pair of variables with the highest correlation. We selected pairs because, as shown by previous studies [27], the higher the correlation, the lower the discriminant validity. For this test, we chose the squared correlation coefficient of resilience and school happiness because they had the highest correlation among variables - and its value was .731, which was lower than the variance distribution mean values (resilience $=.977$, school happiness $=.953$ ) of resilience and school happiness. Thus, we assumed that the measures had appropriate discriminant validity.

\subsection{Data Analysys Method And Process}

The survey proceeded as follows. After the researchers contacted the participants' schools in advance, explained the purpose of the study, and obtained approval and informed consent, they visited the schools, explained the research purpose to the students. And interested participants completed the self-report questionnaires. Also, the study was 
conducted after obtaining consent from the research participants themselves and their parents. The collected data were analyzed using SPSS 18.0 and AMOS 18.0. First, frequency analyses were conducted to examine the demographic characteristics of the samples. Second, Cronbach's $\alpha$ coefficients were calculated to verify the reliability of the measures. Third, a confirmatory factor analysis was conducted to verify the validity of the measurement tools. Fourth, descriptive statistics were calculated to describe and summarize the features of self-esteem, resilience, and school happiness. Fifth, correlation analysis was performed to analyze the correlation between the variables. Sixth, multiple regression analysis was conducted to analyze the effects of subjects' self-esteem on resilience and school happiness.

\section{Result}

\subsection{Descriptive Statistics}

As for descriptive statistics (mean, standard deviation, skewness, kurtosis), we analyzed the total scores of self-esteem, resilience and school happiness, and of all their subfactors, as shown in [Table 5]. The means ranged from 2.49 to 4.18 , and the standard deviation ranged from .61 to .95 . Next, the absolute value of skewness was distributed from .01 to .65 , and the absolute value of kurtosis was distributed from .06 to .58. Previous studies have shown that, for univariate normality tests to prove the normal distribution of the variables, skewness values should be $< \pm 3.0$ [28], and kurtosis values should be $< \pm 10.0$ [29]. Our results showed that all variables met these criteria, so they proved their normal distribution.

Table 5 : Descriptive Statistics Analysis

\begin{tabular}{|c|c|c|c|c|c|}
\hline \multicolumn{2}{|l|}{ Variables } & \multirow{2}{*}{$\begin{array}{r}\text { Mean } \\
4.18\end{array}$} & \multirow{2}{*}{\begin{tabular}{|l|}
$\begin{array}{l}\text { Standard } \\
\text { Deviation }\end{array}$ \\
\\
\end{tabular}} & \multirow{2}{*}{$\begin{array}{r}\text { Skewness } \\
-.65\end{array}$} & \multirow{2}{*}{$\begin{array}{r}\text { Kurtosis } \\
.09\end{array}$} \\
\hline Self- & Positive Self-Esteem & & & & \\
\hline esteem & Negative Self-Esteem & 2.49 & .792 & .46 & .06 \\
\hline \multirow{3}{*}{ Resilience } & Sociality & 3.81 & .672 & .26 & -.52 \\
\hline & Positivity & 3.96 & .666 & -.17 & -.58 \\
\hline & Control & 4.01 & .612 & -.09 & -.48 \\
\hline \multirow{5}{*}{$\begin{array}{l}\text { School } \\
\text { Happiness }\end{array}$} & $\begin{array}{l}\text { Student-Teacher } \\
\text { relationship }\end{array}$ & 3.73 & .897 & $-/ 42$ & -.20 \\
\hline & Self-efficacy & 3.65 & .845 & .01 & -.49 \\
\hline & Environmental satisfaction & 3.64 & .896 & -.13 & -.53 \\
\hline & Learning activity pleasure & 3.61 & .954 & -.28 & -.42 \\
\hline & Psychological stability & 2.90 & .933 & .32 & -.26 \\
\hline
\end{tabular}

\subsection{Correlation Analysis}

Results of correlation analyses for self-esteem, resilience, and school happiness are shown in [Table 6]. They showed that the subfactors of self-esteem, resilience, and school happiness had statistically significant positive or negative correlations. First, there was a statistically significant positive correlation between positive self-esteem and all subfactors of resilience and school happiness, while negative self-esteem had a statistically significant negative correlation with all other variables. Further, all subfactors of resilience were found to have a statistically significant positive correlation with all subfactors of school happiness. Particularly, the correlation between self-efficacy and learning activity pleasure (.831) was the highest, whereas the correlation between friendship and psychological stability (.343) was the lowest. There was no multicollinearity problem because the correlation coefficient between all variables did not exceed .90 .

Table 6: Results of Correlation Analyses among Studied Variables

\begin{tabular}{|l|l|l|l|l|l|l|l|l|l|l|l|l|l|}
\hline \multicolumn{2}{|l}{ Variables } & M & SD & Aa & Ab & Ba & Bb & Bc & Ca & Cb & Cc & Cd & Ce \\
\hline \multirow{2}{*}{$\mathrm{A}$} & $\mathrm{Aa}$ & 4.18 & .71 & 1 & & & & & & & & & \\
\hline & $\mathrm{Ab}$ & 2.18 & .91 & $-.700^{*}$ & 1 & & & & & & & & \\
\hline \multirow{3}{*}{$\mathrm{B}$} & $\mathrm{Ba}$ & 3.81 & .67 & $.656^{* *}$ & $-.587^{* *}$ & 1 & & & & & & & \\
\hline & $\mathrm{Bb}$ & 3.95 & .66 & $.735^{*^{* *}}$ & $-.648^{* *}$ & $.689^{* *}$ & 1 & & & & & & \\
\hline & $\mathrm{Bc}$ & 4.00 & .61 & $.679^{* *}$ & $-.546^{* *}$ & $.660^{* *}$ & $.637^{* *}$ & 1 & & & & & \\
\hline \multirow{3}{*}{$\mathrm{C}$} & $\mathrm{Cb}$ & 3.73 & .89 & $.616^{* *}$ & $-.514^{* *}$ & $.656^{* *}$ & $.620^{* *}$ & $.549^{* *}$ & 1 & & & & \\
\hline & $\mathrm{Cc}$ & 3.65 & .84 & $.637^{* *}$ & $-.515^{* *}$ & $.764^{* *}$ & $.666^{* *}$ & $.617^{* *}$ & $.719^{* *}$ & 1 & & & \\
\hline & $\mathrm{Cd}$ & 3.63 & .89 & $.649^{* *}$ & $-.541^{* *}$ & $.680^{* *}$ & $.700^{* *}$ & $.584^{* *}$ & $.774^{* *}$ & $.758^{* *}$ & 1 & & \\
\hline & $\mathrm{Ce}$ & 3.60 & .95 & $.620^{* *}$ & $-.548^{* *}$ & $.734^{* *}$ & $.698^{* *}$ & $.542^{* *}$ & $.736^{* *}$ & $.831^{* *}$ & $.771^{* *}$ & 1 & \\
\hline & $\mathrm{Cf}$ & 2.87 & 1.06 & $.516^{* *}$ & $-.577^{* *}$ & $.557^{* *}$ & $.560^{* *}$ & $.410^{* *}$ & $.471^{* *}$ & $.469^{* *}$ & $.501^{* *}$ & $.500^{* *}$ & 1 \\
\hline
\end{tabular}

$※$ Correlation is significant at $\mathrm{p}<.01$ (2-tailed).

$※ * * \mathrm{p}<.001 ※ \mathrm{~A}=$ Self-esteem; Aa=Positive Self-esteem; $\mathrm{Ab}=$ Negative Self-esteem, $\mathrm{B}=$ Resilience; $\mathrm{Ba}=\mathrm{Control} ; \mathrm{Bb}=$ Positivity; $\mathrm{Bc}=$ Sociality, $\mathrm{C}=$ School happiness; $\mathrm{Ca}=$ Student-Teacher Relationship; $\mathrm{Cb}=$ Selfefficacy; $\mathrm{Cc}=$ Environmental satisfaction; $\mathrm{Cd}=$ Learning activity pleasure; $\mathrm{Ce}=$ Psychological stability

\subsection{Multiple Regression Analysis}

Results of multiple regression analysis on the influence of self-esteem on resilience in our sample are shown in [Table 7].

Table 7 : Influence of Self-esteem on Resilience

\begin{tabular}{|l|l|l|l|l|l|l|}
\hline $\begin{array}{l}\text { Dependent } \\
\text { Variables }\end{array}$ & $\begin{array}{l}\text { Independent } \\
\text { Variables }\end{array}$ & S.E. & $\beta$ & $\mathbf{t}$ & $\boldsymbol{p}$ & $\begin{array}{l}\text { Tolerance } \\
\text { limits }\end{array}$ \\
\hline \multirow{5}{*}{ Control } & Constant & .348 & & 6.640 & $.000^{* *}$ & \\
\cline { 2 - 7 } & $\begin{array}{l}\text { Positive Self- } \\
\text { Esteem }\end{array}$ & .063 & .482 & 7.232 & $.000^{* *}$ & .511 \\
\cline { 2 - 7 } & $\begin{array}{l}\text { Negative Self- } \\
\text { esteem }\end{array}$ & .049 & -.250 & -3.745 & $.000^{* * *}$ & .511 \\
\cline { 2 - 7 } & $\mathrm{R}(\mathrm{a})=.680 \mathrm{R}^{2}=.463$ adj $\mathrm{R}^{2}=.458 \mathrm{~F}=102.029 \mathrm{p}=.000$ Durbin-Watson $=1.830$ \\
\hline
\end{tabular}




\begin{tabular}{|l|l|l|l|l|l|l|}
\hline \multirow{5}{*}{ Positivity } & Constant & .307 & & 7.260 & $.000^{* *}$ & \\
\cline { 2 - 7 } & $\begin{array}{l}\text { Positive Self- } \\
\text { Esteem }\end{array}$ & .055 & .551 & 9.306 & $.000^{* *}$ & .511 \\
\cline { 2 - 7 } & $\begin{array}{l}\text { Negative Self- } \\
\text { esteem }\end{array}$ & .043 & -.263 & -4.436 & $.000^{* *}$ & .511 \\
\cline { 2 - 7 } & $\mathrm{R}(\mathrm{a})=.759 \mathrm{R}^{2}=.575$ adj $\mathrm{R}^{2}=.572 \mathrm{~F}=160.597 \mathrm{p}=.000$ & Durbin-Watson $=2.191$ \\
\hline \multirow{5}{*}{ Sociality } & Constant & .314 & & 6.755 & $.000^{* *}$ & \\
\cline { 2 - 7 } & $\begin{array}{l}\text { Positive Self- } \\
\text { Esteem }\end{array}$ & .057 & .582 & 8.806 & $.000^{* *}$ & .511 \\
\cline { 2 - 7 } & $\begin{array}{l}\text { Negative Self- } \\
\text { esteem }\end{array}$ & .044 & -.139 & -2.095 & $.037^{*}$ & .511 \\
\hline & $\mathrm{R}(\mathrm{a})=.686 \mathrm{R}^{2}=.471$ adj $\mathrm{R}^{2}=.466 \mathrm{~F}=105.489 \mathrm{p}=.000$ Durbin Watson $=1.918$ \\
\hline
\end{tabular}

$\star \star \mathrm{p}<.01, * \mathrm{p}<.05$

Results showed that all subfactors of self-esteem affected those of resilience: First, positive self-esteem had a statistically significant positive effect on control $(\beta=.482, p<.001)$, while negative self-esteem had a statistically negative effect on control $(\beta=-.250$, $\mathrm{p}<.001$ ), and self-esteem showed an R2 value of .463 when predicting control.

Furthermore, positive self-esteem had a statistically significant positive effect on positivity $(\beta=.551, p<.001)$, while negative self-esteem had a statistically significant negative effect on positivity $(\beta=-.263, p<.001)$, and self-esteem indicated an R2 value of .575 when predicting positivity.

Finally, positive self-esteem had a statistically significant positive effect on sociality $(\beta=.582$, $\mathrm{p}<.001$ ), while negative self-esteem had a statistically significant negative effect on sociality $(\beta=-.139$, $\mathrm{p}<.05$ ), and self-esteem showed an R2 value of .471 when predicting sociality.

Table 8 : Influence of Self-esteem on School Happiness

\begin{tabular}{|c|c|c|c|c|c|c|}
\hline $\begin{array}{l}\text { Dependent } \\
\text { Variables }\end{array}$ & $\begin{array}{l}\text { Independent } \\
\text { Variables }\end{array}$ & S.E. & $\beta$ & $\mathbf{t}$ & $p$ & $\begin{array}{l}\text { Tolerance } \\
\text { limits }\end{array}$ \\
\hline \multirow{4}{*}{$\begin{array}{l}\text { Student- } \\
\text { Teacher } \\
\text { relationship }\end{array}$} & Constant & .494 & & 2.923 & $.004 * *$ & \\
\hline & Positive Self-esteem & .089 & .502 & 7.082 & $.000^{* *}$ & .511 \\
\hline & $\begin{array}{l}\text { Negative Self- } \\
\text { esteem }\end{array}$ & .070 & -.163 & -2.299 & $.022^{* *}$ & .511 \\
\hline & \multicolumn{6}{|c|}{$\mathrm{R}=.627 \mathrm{R}^{2}=.393$ adj $\mathrm{R}^{2}=.387 \mathrm{~F}=76.578 \mathrm{p}=.000$ Durbir Watson $=1.759$} \\
\hline \multirow{4}{*}{ Self-efficacy } & Constant & .457 & & 2.713 & $.007 * *$ & \\
\hline & Positive Self-esteem & .083 & .542 & 7.797 & $.000^{* *}$ & .511 \\
\hline & $\begin{array}{l}\text { Negative Self- } \\
\text { esteem }\end{array}$ & .065 & -.136 & -1.954 & .052 & .511 \\
\hline & \multicolumn{6}{|c|}{$\mathrm{R}=.644 \mathrm{R}^{2}=.415$ adj $\mathrm{R}^{2}=.410 \mathrm{~F}=84.128 \mathrm{p}=.000$ DurbirWatson=1.874 } \\
\hline \multirow{4}{*}{$\begin{array}{l}\text { Environmental } \\
\text { satisfaction }\end{array}$} & Constant & .476 & & 2.562 & $.011^{*}$ & \\
\hline & Positive Self-esteem & .086 & .530 & 7.756 & $.000^{* *}$ & .511 \\
\hline & $\begin{array}{l}\text { Negative Self- } \\
\text { esteem }\end{array}$ & .067 & -.170 & -2.495 & $.013^{*}$ & .511 \\
\hline & \multicolumn{6}{|c|}{$\mathrm{R}=.660 \mathrm{R}^{2}=.436$ adj $\mathrm{R}^{2}=.431 \mathrm{~F}=91.507 \mathrm{p}=.000$ Durbir Watson $=1.707$} \\
\hline \multirow{4}{*}{$\begin{array}{l}\text { Learning } \\
\text { activity } \\
\text { pleasure }\end{array}$} & Constant & .518 & & 2.942 & $.004 * *$ & \\
\hline & Positive Self-esteem & .094 & .464 & 6.639 & $.000^{* *}$ & .511 \\
\hline & $\begin{array}{l}\text { Negative Self- } \\
\text { esteem }\end{array}$ & .073 & -.224 & -3.201 & $.002^{* *}$ & .511 \\
\hline & \multicolumn{6}{|c|}{$\mathrm{R}=.640 \mathrm{R}^{2}=.410$ adj $\mathrm{R}^{2}=.405 \mathrm{~F}=82.289 \mathrm{p}=.000$ Durbir-Watson $=1.984$} \\
\hline \multirow{4}{*}{$\begin{array}{l}\text { Psychological } \\
\text { stability }\end{array}$} & Constant & .600 & & 4.294 & $.000^{* *}$ & \\
\hline & Positive Self-esteem & .108 & .221 & 3.030 & $.003^{* *}$ & .511 \\
\hline & $\begin{array}{l}\text { Negative Self- } \\
\text { esteem }\end{array}$ & .085 & -.422 & -5.793 & $.000^{* *}$ & .511 \\
\hline & \multicolumn{6}{|c|}{$\mathrm{R}=.598 \mathrm{R}^{2}=.357$ adj $\mathrm{R}^{2}=.352 \mathrm{~F}=65.891 \mathrm{p}=.000$ Durbin-Watson $=1.969$} \\
\hline
\end{tabular}

$\star * \mathrm{p}<.01, * \mathrm{p}<.05$
The results of multiple regression analysis to examine the influences of self-esteem on school happiness in our sample are shown in [Table 8].

First, positive self-esteem had a statistically significant positive effect on student-teacher relationship $(\beta=.502, \mathrm{p}<.001)$, while negative selfesteem had a statistically significant negative effect on student-teacher relationship $(\beta=-.163, \mathrm{p}<.05)$, and self-esteem showed an R2 value of .393 when predicting student-teacher relationship.

Next, positive self-esteem had a statistically significant positive effect on self-efficacy $(\beta=.542$, $\mathrm{p}<.001$ ), while negative self-esteem did not have any significant effect on self-efficacy $(\beta=-.136, p>.05)$, and self-esteem showed an R2 value of .415 when predicting self-efficacy.

Further, positive self-esteem had a statistically significant positive effect on environmental satisfaction $(\beta=.530, \mathrm{p}<.001)$, while negative selfesteem had a statistically significant positive effect on environmental satisfaction $(\beta=-.170, p<.05)$, and selfesteem indicated an R2 value of .436 when predicting environmental satisfaction.

Additionally, positive self-esteem had a statistically significant positive effect on learning activity pleasure $(\beta=.464, \mathrm{p}<.001)$, while negative self-esteem had a statistically significant negative effect on learning activity pleasure $(\beta=-.224, p<.01)$, a n d s e lf - e s te e m showed a n R 2 valueof.410whenpredictinglearningactivitypleasure.

Finally, positive self-esteem had a statistically significant positive effect on psychological stability $(\beta=.221, p<.01)$, while negative self-esteem showed a statistically significant negative effect on psychological stability $(\beta=-.422, p<.001)$, and selfesteem showed an R2 value of .357 when predicting psychological stability.

\section{Discussion}

This research aimed to verify the effects of selfesteem on resilience and school happiness among School students participating in school sports clubs. First, positive self-esteem was found to have a positive effect on all subfactors of resilience (control, positivity, and sociality), while negative self-esteem negatively affected these same subfactors. These findings indicated that students' self-esteem was 
closely related to resilience, and that the higher the positive self-esteem, the higher the resilience, and the higher the negative self-esteem, the lower the resilience. Corroborating, [13] conducted a study on students' suicidal thoughts during middle- and highschool; results showed that self-esteem and resilience had the highest correlation, suggesting that selfesteem is the primary variable for resilience. Additionally, [30] indicated that the personal factors affecting university students' resilience were grade, self-efficacy, and self-esteem; they also showed that higher self-esteem can bring higher resilience. In further corroboration, a study by [31] among first- and second-year students in academic and occupational School showed that those with higher self-esteem showed higher resilience to adapt to external environmental changes, suggesting that self-esteem profoundly affected resilience. According to [32], academic School students' self-esteem is a crucial factor in lowering suicidal thoughts, and students' higher positive self-esteem closely relates to higher control, positivity, sociality, and problem-solving ability. Two previous studies [33-34] also suggested that higher self-esteem closely relates to higher resilience.

Thus, it may be implied that higher positive selfesteem leads to higher control, positivity, and problem-solving ability, especially when students face frustrations and failures or stressful situations; this denotes that they try to see the bright sides in the situations, and, after finding the causes of their problems, they make efforts to solve them with others' help. These qualities could help students cope adequately with several unexpected problems that show up during their school time because, even if something eventually was to not go or work the way they wanted it to, students would still try to challenge these problems head on as they have faith in themselves. This goes to show that students should try - and receive external efforts toward - raising their self-esteems because there are several evidences ours included - pointing toward it as being the main influencing factor of resilience. As abovementioned, sports activities are one of the most important factors for building confidence, and confidence is one of the pillars for building self-esteem. Through such a rationale, we do believe that school sports clubs would provide students with good ways to increase their selfesteem, particularly given the fact that, in Korea, students face difficulties in enjoying leisure sports activities outside their school time owing to problems related to the examination-oriented educational system.
Second, regarding the effects of self-esteem on school happiness, positive self-esteem had positive effects on all subfactors of school happiness (friendship, student-teacher relationship, self-efficacy, environmental satisfaction, learning activity pleasure and psychological stability), and negative self-esteem had negative effects on all variables aside from friendship and self-efficacy, upon which it had no significant effects. These findings indicated that, in our sample, students' self-esteem was closely related to school happiness; that higher positive self-esteem brought higher school happiness; and that higher negative self-esteem partially brought lower school happiness. That is, the more students evaluate themselves positively, the more they consider themselves valuable, the more they avoid comparing themselves with others, and the more happiness they feel in school-because they tend to have positive friendships and student-teacher relationships, higher rates of self-efficacy and environmental satisfaction, more learning activity pleasure, and higher levels of psychological stability.

These abovementioned results partially agree with previous studies: one suggested that the improvement of positive self-esteem leads to higher school happiness, and that self-esteem is highly related to happiness [12]. Other studies showed that, the higher the self-esteem, the happier people feel because they experience more positive than negative emotions and are more stable [35-38]. Our results also emphasized that, among School students' characteristics, selfesteem may be a major reinforcing factor when trying to lower suicidal thoughts, an idea corroborated by the research results of [32], whom advocated for the need of providing education that fosters students' positive self-concept and self-esteem. Corroborating, [10] found that School students with positive self-esteem were better adapted to school life, indicating that selfesteem closely relates to school life adaptation; such a finding demonstrates a similar relationship to that between self-esteem and school happiness in other aforementioned studies. Additionally, [39] found that the most influential variable among university students' characteristics regarding adaptation to school life was their self-esteem, highlighting its importance related to school life. Another study by [40] among university students participating in convergent leisure sports activities found that selfesteem had a positive effect on their adaption to university life, once more supporting our research findings - self-esteem plays a crucial role in school life. 
These findings suggest that high self-esteem can positively influence School students to create a positive self-image by themselves, to build confidence, and to be responsible during their studies and toward relationships with others [41-42], which suggests that high self-esteem leads to happiness in their school lives. Moreover, our results also suggest that the formation and development of positive selfesteem is an important leading variable for School students' school happiness. Therefore, various educational efforts are needed and should be taken to allow for such students to adequately and happily adjust to school life. Participating in school sports clubs can be a great program for them to enjoy their time at school without any pressure, and since it has been shown to be one of the most important activities for building confidence in school, it may also be a basis for building self-esteem [43-44]. Thus, we believe that policy makers and school managers should consider introducing a school sports club program in the School curriculum, so that students can enjoy their leisure time and increase their school happiness.

Notwithstanding, our results also showed that negative self-esteem did not have a significant effect on the friendship nor on the self-efficacy subscales of school happiness. Thus, it can be assumed that School may be a period when students cherish their friendship the most, feel common stress owing to specific situations (such as university entrance exams and entrance into the work market), form a consensus, and rely on their friends. Furthermore, since there are insufficient studies to support the relationship between negative self-esteem and self-efficacy, we believe future studies are warranted to examine this specific relationship to provide more empirical evidence toward its existence.

\section{Conclusion And Suggestions}

This research analyzed the effects of self-esteem on resilience and school happiness among School students participating in school sports clubs, and provided basic data on the role of school sports clubs in the promotion of school happiness and how they can be used for this purpose. We performed this study with the intent to help develop research and education programs that improve students' school happiness. For this purpose, this research targeted 324 School students who participated in school sports clubs.

In our sample, there was a significant positive correlation between positive self-esteem and all subfactors of resilience and school happiness, and negative self-esteem had a negative correlation with all other variables. And, positive self-esteem had a positive influence on all subfactors of resilience (control, positivity, and sociality), and negative selfesteem had a negative influence on all subfactors of resilience. Additionally, positive self-esteem positively affected all subfactors of school happiness (student-teacher relationship, self-efficacy, environmental satisfaction, learning activity pleasure, and psychological stability), while negative selfesteem negatively affected some subfactors of school happiness (student-teacher relationship, environmental satisfaction, learning activity pleasure, and psychological stability). Finally, regarding the resilience subscales and their influence on school happiness, control had a positive influence on all its subfactors, except for friendship; positivity had a positive effect on all subfactors; and sociality had a positive effect on friendship and self-efficacy.

This study also had many limitations. First, since the samples were composed of School students living in some specific areas of Korea, our collected data was partially biased owing to sample- and region-related tendencies; future studies should expand the geographical scope of their samples and select more diverse participants, so that the findings can be further generalized. We also suggest taking into account various age groups and personal characteristics.

Second, regarding research outcomes, there are limitations in presenting empirical data on whether or not school sports clubs indeed contribute to building self-esteem and resilience; therefore, to help with the expansion and diversification of school sports clubs, we think future studies should analyze their influence through comparative and analytical designs after dividing the sample into participants and nonparticipants of these clubs.

\section{References}

[1] Yoo, J., Kim, B. M., Shin, H. S., Jeong, J. Y., \& Choi, N. H. (2010). Relationship with Stress from University Entrance Competitions, Self Esteem, Coping Strategy of School Students in Korea. Crisisonomy, 6(3), 223-241.

[2] Kim, H. W., \& Hong, M. A. (2007). Exploration of the Extents and Factors Explaining Well-Being among Korean Adolescents. Korean Journal of 
Youth Studies, 14(2), 269-297.

[3] Jun, M. K., \& Jang, J. S. (2009). A Longitudinal Study of the Development of Happiness during Childhood. Family and Environment Research, 47(3), 103-118.

[4] Song, Y. M. (2011). The Effects of the special purpose highschool students' parent-child relationship, ego-resilience and study competence on school happiness. Korean Journal of Educational Psychology. 25(3). 647669.

[5] Kim, J. B., \& Kim, T. E. (2008). The Development and Validation of Korean School Happiness Scale. The Korean Journal of Education Psychology, 22(1), 259-279.

[6] Kim, S. Y., \& Cho, G. P. (2016). The Effect of Academic Failure Tolerance, Psychological Growth Environment, and Social Support of Middle School Students on Their School Happiness. Journal of Learner-Centered Curriculum and Instruction, 16(2), 259-279.

[7] Moon, J. S., \& Choi, G. J. (2017). The Effects of Physical Self-Concept and Social Skills of Middle School Students Participating in School Sports Club on School Happiness. The Korean Journal of Sport, 15(3), 119-128.

[8] Ministry of Education (2016). Plans to promote school sports in 2016.

[9] Yook, Y. S. (2015). The effects of the after-school sports activities on self-esteem, exercise emotion, and adaptation to school life for the early adolescents. The Korean Society of Sports Science, 24(5), 739-749.

[10] Choi, S. B., \& Kim, D. I. (2011). The relationship between family strengths, self esteem and school life adjustment of sports participant by highschool students. The Korean Journal of Sport, 9(1), 167-178.

[11] Zuzanek, J., J. Robinson. \& Yoshi Iwasaki. (1998). The relationship between stress, health, and physically active leisure as function of lifecycle. Leisure Science, 20, 253-275.

[12] Koo, J. S. (2009). Does Happiness Build
Psychological Resources?. Korea Journal of Social and Personality Psychology, 23(1), 165179.

[13] Yeo, J. Y., \& Park, T. Y. (2013). A Study of Adolescent's Suicidal Ideation: The Mediation Effect of Resilience and Self-Esteem. Korean journal of youth studies, 20(8), 121-145.

[14] Kim, H. T. (2012). Factors Influencing Resilience of Group Home Children - Using Self-esteem as Mediator - . Journal of the Korean society of child welfare, 38, 211-234.

[15] Kim, J. H. (2011). Resilient elasticity: A delightful secret that turns ordeal into good fortune. Gyunggi: Wisdom House Press.

[16] Park, N. J., Lim, N. Y., \& Lee, C. H. (2018). The Effect of Resilience in Career Decision-Making among Specialized Technical School Students. Journal of the Korean Institute of industrial educators, 43(1), 20-40.

[17] Kim, I. H. (2015). The Influences of the Youth' Empathy and Resilience on Violent Attitudes and Problematic Behaviors in School Sports Clubs Participation. Korean Journal of Youth Studies, 22(4), 23-48.

[18] Bea, J. S., Joeng, H. C., \& Won, D. Y. (2015). The influence of 'Saturday Sports Day' Participation Experience on Middle School Students' resilience and School Adjustment. Korean Journal of Sport Studies, 54(5), 415-424.

[19] Kim, S. W., \& Lee, D. H. (2015). The Influence of Physical Self-concept of Middle School Sports Club Participants on Resilience and School Happiness. Journal of Sport and Leisure Studies, 62, 563-574.

[20] Jun, B. J. (1974). Self-Esteem: A test of it's Measurability. collection of writings Yonsei, 11, 107-129.

[21] Rosenberg, M. (1965). Society and the adolescent self-image. Princeton, NJ : Princeton University Press.

[22] Duran, R. L. (1983). Communicative adaptability: A measure of social communicative competence. Communication 
Quarterly, 31, 320-326.

[23] Shin, W. Y., Kim, M. G., \& Kim, J. H. (2009). Developing Measures of Resilience for Korean Adolescents and Testing Cross, Convergent, and Discriminant Validity. Korean Journal of Youth Studies, 20(4), 105-131.

[24] Seligman, M. E. (2004). Authentic happiness: Using the new positive psychology to realize your potential for lasting fulfillment. Simon and Schuster.

[25] Cho, C. H. (2016). Application of SPS/AMOS: Statistic Analysis of Structural Equation Model. Seoul: Chungram Press.

[26] Hong, S. H. (2000). The criteria for selecting appropriate fit indices in structural equation modeling a nd $t$ heir $r$ ationales. Korean Journal of Clinical Psychology, 19(1), 161-177.

[27] Woo, J. P. (2015). Concept and Understanding of Structural Equations. Seoul: Hannarae Press.

[28] West, S. G., Finch, J. F., \& Curran, P. J. (1995). Structural equation model with non-normal variables: Problems and remedies. In R. Hoyle (Ed.), Structural Equation Modeling: Concepts, Issues and Applications. Newbury Park, CA: Sage.

[29] Kline, R. B. (2011). Principles and practice of structural equation modeling(3nd ed.). New York: Guilford.

[30] Son, D. S., \& Moon, Y. H. (2011). A Study on the influence factors the resilience of social welfare majors in college. Korean Journal of Social Welfare Education, 16, 48-69.

[31] Kim, K. S., \& Kim, H. K. (2011). A Study on Relation Between Perceived Parental Rearing Attitude and Self-esteem and Ego-resilience of School Students. Educational Research, 19(2), 143-170.

[32] Seo, H. S. (2006). A study on the factors influencing the suicide ideation of juveniles: focused on psychosocial factors. Doctor's thesis, Deagu University, Deagu, Korea.

[33] Rutter, M. (1987). Psychosocial resilience and protective mechanisms. American Journal of
Ortho psychiatry, 57(3), 316-331.

[34] Kim, K. C., Kim, E. O., \& Kim, E. H. (2017). The effect of self-esteem and resilience of preservice early childhood education teachers on educational beliefs. Asia-pacific Journal of Multimedia Services Convergent with Art, Humanities, and Sociology, 7(10), 229-238.

[35] Choi E. Y., Choi, I. R. (2002). A Study on the Relation Between Self Esteem and Mental Health in Middle School Students. A Study on the Relation Between Self Esteem and Mental Health in Middle School Students, 15(1), 63-72.

[36] Kang, K. J., \& Jeong, E. M. (2003). The Effect of a Self-Growth Group Counseling Program to Improve Self-Esteem on Children of Group Home. Family and Environment Research, 41(11), 117-133.

[37] Park, H. S., \& Moon, S. Y. (2014). Mediator Effect of School Life Adaptation Affecting Relationships Between University Students' Happiness, Self-Respect and Social Support. Korean Journal of Local Government \& Administration Studies, 28(3), 197-218.

[38] Jung, S. H., Park, S. J., \& Jang, H. S. (2016). Stress, Self-esteem and School Adaptation of Middle School Students from Low Income Class based on Obesity. Journal of Research in Curriculum Instruction, 20(6), 490-499.

[39] Lee, J. H. (2006). A Study on the Effects of Family Strengths and Self-esteem on the Adaptation in School Life for College Students. Master's thesis, Deagu University, Deagu, Korea.

[40] Han, S. J., \& Lee, S. H. (2015). A Study on Structural Relations among the Social Support, Self-Esteem, and College Life Adjustment of College Students Participating in Convergence Leisure Sports Activities. Journal of Digital Convergence, 13(8), 515-523.

[41] Song, H. H., Song, J. H., \& Lee, S. M. (2020) Resilience and School Happiness Through Participation of School Students in the School Sports Club. International Journal of Social Welfare Promotion and Management, 7(2), 1724. 
[42] Sin, J. G., Park, J.L., Choi, J. H. (2018). The Relationship between Participation in Afterschool Sports Club, Academic Emotional Regulation and Self-Efficacy of Middle School Students.International Journal of Child Warfare, 2(2), 7-12.

[43] Jang, H. A., Choi, J. H., Park. J. L. (2018). The Effect of Middle School Students` Participation in Saturday Sports on Physical Self-Efficacy and Self-Directed Learning Ability.International
Journal of Child Warfare Promotion and Management, 2(2), 13-18.

[44] Kim, S. K., Kim, M. J., \& Yoo, J. I. (2016). The Effects of Sports Values on Sports Motivation and Exercise Satisfaction among participants.International Journal of IT-based Social Welfare Promotion and Management. 3(1), 151-156. 